\title{
Shimstocks and Their Vital Role in Prosthodontics and Restorative Dentistry
}

Accurate occlusal contacts are critical in prosthodontics and restorative dentistry. Minor changes in occlusal contacts usually are tolerated due to periodontal ligaments and soon will be re-established by their proprioceptive feedback. However, any occlusal interference or a prematurity cannot be overlooked and underestimated due to dynamic nature of dental occlusion. Moreover in dental implant restorations, due to lack of periodontal ligament, the occlusal forces directly transmit from the occlusal surfaces of the restorations to the bone-implant inter-phase leading to detrimental force-dynamics in implant therapy. Hence careful evaluation of occlusal contacts before and after the restorations or prosthesis

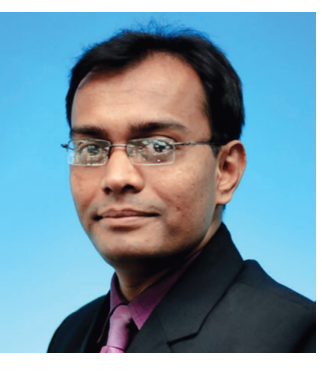
being placed is a vital clinical step. As a restorative dentist we should always evaluate the occlusal contacts before a tooth (or teeth) is prepared. Then ensure this is re-established when the provisional restoration has been fitted, when assessing the laboratory made restoration on the master model (against the opposing model), and once the restoration has been cemented in the mouth. Several methods are in practice using articulating papers and the shimstocks or milar strips to dignose and correct the occlusal interferences. Mylar strips or shimstocks are the usually 8 micron thick strips. The researchers and clinicians have different opinions regarding the utility of using these methods to correct the occlusal interferences.

Anderson et $\mathrm{al}^{1}$ reported on the reliability of dentists' ability to evaluate occlusal contacts in the intercuspal position. Shimstock and an articulating paper were compared in the evaluation of occlusal contacts of 337 antagonist occlusal pairs in 24 young adults by two examiners. The shimstock displayed better reliability than articulating film and appeared suitable for clinical measurement of occlusal contacts in intercuspal position. Gupta et $\mathrm{al}^{2}$ evaluated the pattern of occlusal contacts in lateral excursion and compared the relative accuracy of shim stock and articulating paper for determining occlusal contact patterns. The patterns of occlusal contacts of 70 young adults were examined in various lateral positions with shimstock and articulating paper in regulated lateral positions $0.5,1,2$, and $3 \mathrm{~mm}$ from the maximum intercuspation. They concluded that the shimstocks exhibit superior accuracy and reliability as compared to the articulating papers. Harper and Setchell ${ }^{3}$ investigated the influence of varying occlusal forces, occlusal contacts, or small gaps ( 0 to 8 microns) on the force required to pull 8 micron shimstock from between a tooth and a restorative material specimen. They concluded that, using 8 micron shimstock, 0-, 2-, and 4-micron gaps cannot be differentiated, a gap of up to 6 microns may be assessed with a tight grip, and even an 8-micron gap may be recorded as a light contact. Carossa et al ${ }^{4}$ evaluated the influence of recording strip thickness and of operator-dependent factors on the assessment of contact area on a cast mounted on an articulator. Thirteen dental students and 13 experts (dentists and dental technicians) performed occlusal maneuvers on casts mounted on an articulator, using recording strips of 8-and 40-micron thickness. The results revealed that the assessment of occlusal contact in the dental laboratory was significantly influenced by paper thickness, operator experience, biting force, and time. Maness ${ }^{5}$ compared the performance of a computerized occlusal analysis (T-Scan) system with that of Accufilm and Shimstock foil for the registration of tooth contacts on a laboratory model and concluded that the traditional methods were similar to the computerized analysis but the T-Scan system provided additional differential diagnostic information in force and time modes for an improved occlusal analysis.

To my knowledge, there is still a lot more scope for further research on this vital issue in day to day clinical practice. As the inaccurate occlusal interferences lead to disturbances in entire stomatognathic system including teeth or implants, jawbones, muscles of mastication and the temporo-mandibular joint. 


\section{References}

1. Anderson GC, Schulte JK, Aeppli DM. Reliability of the evaluation of occlusal contacts in the intercuspal position. J Prosthet Dent 1993;70:320-3.

2. Gupta A, Shenoy VK, Shetty TB, Rodrigues SJ. Evaluation of pattern of occlusal contacts in lateral excursion using articulating paper and shim stock: An in vivo study. J Interdiscip Dent 2013;3:109-13.

3. Harper KA, Setchell DJ. The use of shimstock to assess occlusal contacts: a laboratory study. Int J Prosthodont. 2002;15(4):347-52.

4. Carossa S, Lojacono A, Schierano G, Pera P. Evaluation of occlusal contacts in the dental laboratory: influence of strip thickness and operator experience. Int J Prosthodont. 2000;13(3):201-4.

5. Maness WL. Laboratory comparison of three occlusal registration methods for identification of induced interceptive contacts. J Prosthet Dent. 1991;65(4):483-7.

Pravinkumar G Patil

Managing Editor

International Journal of Prosthodontics and Restorative Dentistry School of Dentistry, International Medical University

Kuala Lumpur, Malaysia 